\title{
Enhanced reheating via Bose condensates
}

\author{
Rouzbeh Allahverdi, ${ }^{1}$ Robert Brandenberger, ${ }^{2,3,4}$ and Anupam Mazumdar ${ }^{4}$ \\ ${ }^{1}$ Theory Group, TRIUMF, 4004 Wesbrook Mall, Vancouver, British Columbia, Canada V6T $2 A 3$ \\ ${ }^{2}$ Physics Department, Brown University, Providence, Rhode Island 02912, USA \\ ${ }^{3}$ Perimeter Institute, Waterloo, Ontario, Canada N2J 2W9 \\ ${ }^{4}$ Physics Department, McGill University, 3600 University Road, Montréal, Quebec, Canada H3A 2T8
}

(Received 28 July 2004; published 29 October 2004)

\begin{abstract}
In supersymmetric extensions of the particle physics standard model, gauge invariant combinations of squarks and sleptons (flat directions) can acquire large expectation values during a period of cosmological inflation. If the inflaton sector couples to matter fields via these flat directions, then new channels for efficient reheating, in particular, via parametric resonance instabilities, are opened up. These can lead to efficient reheating induced by the flat directions even if the bare coupling constants are small. In this Letter we discuss various channels which yield this "enhanced reheating" effect, and we address some cosmological consequences.
\end{abstract}

DOI: 10.1103/PhysRevD.70.083535

PACS numbers: $98.80 . \mathrm{Cq}, 11.30 . \mathrm{Pb}$

\section{INTRODUCTION}

There are strong indications that the early Universe might have undergone a phase of inflationary expansion [1]. The success of big-bang cosmology puts severe constraints on early Universe cosmology, and therefore, if inflation is the right paradigm, then it must yield a way of understanding the origin of all observed matter, including the baryons of the standard model (SM). In spite of many attempts, it has proven hard to pin down the inflaton sector. Since its couplings with standard model fields in the bare Lagrangian are constrained to be very small, it is usually assumed that the inflaton is a SM gauge singlet, and in most models it is even considered as an absolute gauge singlet (for reviews, see, e.g., [2,3]). This leads to the question of how the Universe reheats and produces the matter we observe today.

It is commonly believed that the inflaton sector couples to either the SM sector or the matter sector of the minimal extension of the SM, the minimal supersymmetric standard model (MSSM) through nonrenormalizable interactions (for a review, see [4]). In this context, however, it is not clear why the inflaton would decay at a sufficient rate into the SM degrees of freedom. This is the issue we will focus on in this Letter. We will concentrate on the case when the inflaton couples to either SM or MSSM fields via interactions which are suppressed by the scale at which new physics is being invoked, which could be either the grand unified theory scale, string scale, or the Planck scale. It turns out that the careful consideration of the evolution of the fields corresponding to the flat directions leads to a new mechanism of reheating which we call "enhanced reheating."

As is well known, MSSM has flat directions, made up of gauge invariant combinations of squarks and sleptons (possibly including right-handed sneutrinos), which may acquire nonvanishing expectation values during inflation (see, e.g., [2] for a review), thereby forming homogeneous condensates. The condensates may play a significant role in many cosmological phenomena (see, e.g., [5] for recent reviews), such as generating the baryon asymmetry, or producing the dark matter particles by first fragmenting into $Q$ balls which then decay [6]. It has also been suggested that the cosmological density perturbations could be due to fluctuations of a MSSM flat direction condensate [7]. In this Letter we will study novel effects of MSSM flat direction condensates for reheating.

\section{ENHANCED PERTURBATIVE REHEATING}

Even if at the level of the bare particle physics Lagrangian there is no coupling between the inflaton and the MSSM matter fields, such a coupling will be generated via gravitational effects. The resulting terms will typically correspond to a nonrenormalizable field theory and will be suppressed by the Planck mass. We will model these terms via a simple superpotential term

$$
W \supset \lambda \frac{\Phi}{M_{\mathrm{P}}} \mathbf{X} \Psi_{1} \Psi_{2}
$$

where $\Phi$ is the (singlet) inflaton superfield, $\mathbf{X}$ is the superfield containing the condensate, $\Psi_{i}$ stands for MSSM matter superfields, $M_{\mathrm{P}}=2.4 \times 10^{18} \mathrm{GeV}$ is the reduced Planck mass, and $\lambda$ is a coefficient. Note that all what is needed is that $\mathbf{X} \Psi \Psi$ be a gauge invariant combination of the MSSM fields. Then, regardless of any discrete or continuous global symmetry (e.g., $R$ symmetry) that the theory may have, gravitational effects are expected to generate the above superpotential term. If the inflaton is a gauge singlet up to $M_{\mathrm{P}}, \lambda \sim \mathcal{O}(1)$ typically. On the other hand, if $\Phi$ is nonsinglet under new physics at a scale $M_{\text {new }}<M_{\mathrm{P}}, \lambda$ will generally be suppressed by powers of $M_{\text {new }} / M_{\mathrm{P}}$.

Around the minimum of its potential, the inflaton superpotential can be approximated by $m_{\phi} \Phi^{2} / 2$. At the end of inflation $\langle X\rangle=X_{I}$ and, treating the inflaton as a 
real scalar field, Eq. (1) results in a Lagrangian of the form

$$
\frac{1}{\lambda}\left(\frac{M_{\mathrm{P}}}{X_{I}}\right) \mathcal{L} \supset \phi \bar{\psi} \psi+m_{\phi} \phi\left(\chi^{2}+\chi^{* 2}\right)+\lambda\left(\frac{X_{I}}{M_{\mathrm{P}}}\right) \phi^{2}|\chi|^{2},
$$

where $\psi$ is a Dirac fermion containing the fermionic components of $\Psi_{1}$ and $\Psi_{2}$, and $\chi$ denotes the scalar components of the $\Psi$ superfields.

A phase of primordial inflation driven by the slowly rolling scalar field $\phi$ sweeps the Universe clean of any particle excitations which might have been present before inflation, but leads to vacuum fluctuations which generically will produce quasihomogeneous expectation values (condensates) of light fields such as $X_{I}$. After the slowroll approximation breaks down, the inflaton field will begin a phase of coherent oscillations about the minimum of its potential. During this phase, the coherent oscillations will dominate the energy density of the Universe. Because of couplings of the inflaton field to other fields, the oscillations will transfer some of their energy density to the matter particles. Upon thermalization of these particle excitations, a radiation-dominated FriedmannRobertson-Walker (FRW) universe will result, as in the hot big-bang model. This process is called reheating. Successful big-bang nucleosynthesis [8] requires that the reheat temperature of the Universe obey $T_{R} \gtrsim \mathcal{O}(\mathrm{MeV})$.

In the initial analyses [9], the energy transfer from the coherent inflaton field to matter was treated perturbatively, using the lowest order analysis to determine the rate $\Gamma_{d}$ with which a single $\phi$ particle at rest decays into quanta of the fields which $\phi$ couple to. While the Hubble constant $H$ exceeds the decay rate, the inflaton will lose energy primarily to the expansion of space, but as soon as $H$ drops $\Gamma_{d}$, the energy of $\phi$ goes primarily into matter particles, resulting in a gas of matter with a temperature $T_{R}$ given by

$$
T_{R} \sim 0.1\left(\Gamma_{d} M_{\mathrm{P}}\right)^{1 / 2} .
$$

In our toy model (2), the inflaton can decay perturbatively via several channels. In the limit that the inflaton mass $m_{\phi}$ is much larger than the mass of $\psi$ and $\chi$, the perturbative decay rate of $\phi$ into fermion and scalar pairs is given by

$$
\Gamma_{I}=\lambda^{2} \frac{m_{\phi}}{4 \pi}\left(\frac{X_{I}}{M_{\mathrm{P}}}\right)^{2} .
$$

Let us assume that the perturbative decay rate in the absence of the $X$ condensate is $\Gamma_{0}$ [with associated reheat temperature $T_{R, 0}$ determined via (3)]. From (4) it immediately follows that a large value of $X_{I}$ leads to enhanced perturbative reheating. The condition for enhanced reheating (i.e., $\Gamma_{I}>\Gamma_{0}$ ) is

$$
\lambda^{2}\left(\frac{X_{I}}{M_{\mathrm{P}}}\right)^{2}>10^{3} \frac{T_{R, 0}^{2}}{m_{\phi} M_{\mathrm{P}}} .
$$

As an example, consider the chaotic inflation model with $m_{\phi}=10^{13} \mathrm{GeV}$ and $T_{R, 0}=10^{9} \mathrm{GeV}$. Then the bound in (5) translates to $\lambda X_{I}>10^{13} \mathrm{GeV}$.

\section{ENHANCED PARAMETRIC REHEATING}

As first discussed in [10] (see also [11]), in many models there is a nonperturbative and much more efficient reheating mechanism which makes use of the parametric resonance instability in the field equation of the particles being produced ( $\psi$ and $\chi$ in our model) in the presence of an oscillating inflaton field. Note that, as first discussed heuristically in [10] and then analytically in $[12,13]$, the instability is present even when taking the expansion of space and the resulting decay of the inflaton oscillation amplitude into account. This mechanism leads to an initially highly nonthermal distribution of particles (including inflatons), while full thermal equilibrium takes much longer to establish (see, e.g., [14] for a recent study).

The Lagrangian (2) contains three separate channels for resonance. The last term on the right-hand side of (2) corresponds to two-particle induced resonant production of $\chi$ bosons and was treated in detail in [15]. The second term leads to single-particle induced resonant production of $\chi$ bosons and was analyzed in [13], while the first term leads to parametric production of $\psi$ fermions and was treated in detail in [16]. The third term dominates for large values of $X_{I}$.

Let us first focus on the third term on the right-hand side of (2). If we neglect the other two terms, then in the presence of the oscillating inflaton field, the equation of motion for $\chi$ will lead to exponentially growing solutions with a Floquet exponent $\mu$ much larger than the Hubble expansion rate. The instability occurs for a large range of comoving momenta (and thus the instability is called of broad resonance type [15]). The instability is effective as long as [15]

$$
\lambda\left(\frac{X_{I}}{M_{\mathrm{P}}}\right) \phi>m_{\phi} .
$$

The effective coupling constant during inflation is bounded by the amplitude of the induced cosmic microwave background (CMB) anisotropies, leading to the constraint

$$
\lambda^{2}\left(\frac{X_{I}}{M_{\mathrm{P}}}\right)^{2} \leq 10^{-6}
$$

making use of the fact that an interaction coupling constant arising in (2) will induce a one-loop correction to the quartic inflaton self-coupling which is bounded by the standard fluctuation analysis (see, e.g., [17] for a review).

During inflation, long wavelength quantum fluctuations of $X$ accumulate in a coherent state with a maximum 
quasihomogeneous field value given by [3] $\left\langle X^{2}\right\rangle=$ $3 H_{I}^{4} / 8 \pi^{2} m_{X}^{2}$, which for condensate masses smaller than the Hubble constant during inflation can be many orders of magnitude larger than $H_{I}$. The exception is when supersymmetry breaking by the inflaton energy density [18] results in a correction $\approx+H_{I}^{2}$ to the $(\text { mass) })^{2}$ of $X$ during inflation. ${ }^{1}$ Another way to obtain a large expectation value $X_{I}$ is if $X$ is exactly massless during inflation and obtains a nonvanishing mass during a phase transition which takes place during the early stages of reheating, in analogy to how the QCD axion field develops an expectation value after the QCD phase transition. Note that for field values larger than $M_{\mathrm{P}}$, gravitational corrections to the potential may not be under control, and hence we will consider $H_{I} \ll X_{I} \leq M_{\mathrm{P}}$ at the end of inflation.

The scenario of enhanced parametric resonance is now as follows. At the end of the slow-rolling phase of inflation, $\phi \sim M_{\mathrm{P}}$, and, since the inflaton mass $m_{\phi}$ is constrained by the amplitude of the CMB fluctuations to satisfy $m_{\phi}<10^{-6} M_{\mathrm{P}}$, the broad resonance condition (6) is satisfied and resonant $\chi$ production occurs. As a consequence of the energy loss to particle production and of the expansion of space, the amplitude of $\phi$ oscillations will decrease. If the condition (6) is satisfied when resonant particle production becomes explosive, inflaton oscillations will decay in the broad resonance regime [15]. When the condition (6) ceases to be satisfied the broad resonant decay shuts off.

Even after the period of broad resonance ends, parametric resonance via the first two terms in (2) will continue for a while. To see this, focus on the resonant production of $\chi$ particles via the single $\phi$ coupling in (2). The perturbative decay rate is the same as the perturbative decay rate (4) into fermions via the first term on the right-hand side of (2). As studied in detail in [13], the parametric resonant decay of $\phi$ proceeds by exciting particles in narrow resonance bands, the first at $k=$ $m_{\phi} / 2$. Decay into the lowest resonance band dominates the overall decay rate, and, focusing on this band, the decay rate is given by

$$
\Gamma_{\chi} \sim \frac{H m_{\phi}^{2}}{16 \pi^{2} \phi^{2}} \sinh ^{2}\left[\lambda^{2}\left(\frac{X_{I}}{M_{\mathrm{P}}}\right)^{2} \frac{\phi^{2}}{H m_{\phi}}\right]
$$

(see Eq. (125) of [13]).

If the argument of the hyperbolic function is smaller than 1 , the result reduces to the perturbative decay rate. Thus, this channel has enhanced parametric reheating provided the argument is larger than 1, which is the case if (taking into account the fact that the inflaton potential is taken to be quadratic)

\footnotetext{
${ }^{1}$ Note that a correction $\approx-H^{2}$ typically results in $X_{I} \gg H_{I}$, since $X$ will in this case settle at the minimum of the potential which is far away from the origin.
}

$$
\lambda^{2} \frac{X_{I}^{2} \phi}{M_{\mathrm{P}} m_{\phi}^{2}}>1
$$

By inserting the saturation values of (6) and (7) into (9), it follows immediately that this condition remains satisfied once the broad resonant decay shuts off, provided that $M_{\mathrm{P}}>10^{3} m_{\phi}$.

Once the value of $H$ drops below $m_{X}$, the $X$ condensate will also begin damped oscillations. Thus, the conditions in (6), respectively (9), may cease to be satisfied before broad, respectively, narrow, resonant decay completes. However, the oscillations of $X$ lead to another channel of parametric reheating, namely, reheating induced by the coupling of $X$ to other matter fields in the part of the Lagrangian which is independent of the inflaton (see [19] for a discussion of this scenario).

To conclude this section, we have demonstrated that large expectation values of scalar condensates such as arise in the MSSM open up several channels for enhanced reheating via parametric resonance.

\section{SOME CONSEQUENCES}

Let us first discuss the consequences of the above analysis for the "temperature" $T_{R}$ after reheating, by which we mean the fourth root of the energy density in matter after the inflaton has lost a majority of its energy into matter. ${ }^{2}$ There are various possible scenarios.

In the first case, the broad resonance condition (6) is satisfied when the inflaton begins to oscillate, which occurs when $\phi \sim M_{\mathrm{P}}$. In this case, the energy transfer into matter happens explosively via broad parametric resonance, yielding the value (remembering again that the potential for $\phi$ is quadratic)

$$
T_{R} \sim\left(m_{\phi} M_{\mathrm{P}}\right)^{1 / 2},
$$

which, for values $m_{\phi} \sim 10^{-6} M_{\mathrm{P}}$ obtained from the amplitude of the observed CMB spectrum under the hypothesis that adiabatic perturbations of the inflaton generate the observed anisotropies, is about $10^{16} \mathrm{GeV}$. This scenario is realized quite naturally if the initial value of the condensate is close to the Planck scale.

If the broad resonance condition (6) is not satisfied when the inflaton starts oscillating, or ceases to be satisfied before broad resonant decay completes, but the condition (9) for effectiveness of the narrow resonance instability via the second interaction term on the righthand side of (2) is satisfied, then once again the energy

\footnotetext{
"If the state after this stage of "preheating" [12] were thermal, then the quantity $T_{R}$ thus computed would indeed be the usual thermodynamical temperature of matter. However, if the reheating proceeds via parametric resonance, then the state of matter immediately after preheating will be far from thermal.
} 
transfer to matter will be immediate and the temperature after the preheating will be given by (10).

The third scenario arises when neither (6) nor (9) are satisfied, but when the perturbative decay rate in the presence of the condensate $\Gamma_{I}$ is larger than $m_{X}$, the mass of the condensate. In this case, reheating completes before $X$ starts its oscillations. In this case, the reheat temperature is given by (3), with $\Gamma_{d}=\Gamma_{I}$, yielding

$$
T_{R} \simeq 3 \times 10^{-2} \lambda X_{I}\left(\frac{m_{\phi}}{M_{\mathrm{P}}}\right)^{1 / 2} .
$$

Finally, if none of the above resonance conditions are satisfied and in addition $\Gamma_{I}<m_{X}$, then the condensate will begin to oscillate once the Hubble parameter $H$ drops below $m_{X}$, and this will lead to new production channels for the production of matter via $X$ decay. Assuming that the decay is rapid on a Hubble time scale, the resulting reheat temperature $T_{R}$ (quotation makes indicating once again that the quantity is simply a measure of the energy density of the matter particles after the decay of the $X$ condensate) will be given by

$$
T_{R} \sim\left(m_{X} X_{I}\right)^{1 / 2},
$$

unless the result is smaller than what is obtained from (11), in which case most of the energy of matter particles results from the inflaton decay which starts once $\Gamma_{I}=H$, i.e., after the condensate has started to oscillate.

We have seen that reheating can be efficient even for very small inflaton couplings to matter. This can have good or bad effects for early Universe cosmology. On the positive side, the enhancement of reheating by the $X$ condensate may make it possible to obtain reheating temperatures sufficiently high for successful baryogenesis and/or dark matter production even when the reheating temperature $T_{R, 0}$ (calculated using the perturbative decay rate in the absence of the condensate) is too low. However, enhanced reheating should not lead to the gravitino problem [20]. If $T_{R}>10^{9} \mathrm{GeV}$, then there will be a gravitino overproduction problem unless the gravitino mass $m_{3 / 2}$ obeys $m_{3 / 2} \geq 20 \mathrm{TeV}$ (this happens, for example, in models of anomaly-mediated supersymmetry breaking [21]). Otherwise, late entropy generation will be required to dilute the excess of gravitinos. As we have seen above, if the parametric resonance channels for reheating are open as a consequence of a large expectation value of $X$, then $T_{R}$ can be of the order of $10^{16} \mathrm{GeV}$ and we will be faced with the gravitino problem. Even if the parametric resonance channels are closed, then, using (11), we conclude that there will be a gravitino problem if

$$
\lambda X_{I}>10^{-5} M_{\mathrm{P}} .
$$

A second stage of inflaton like in thermal inflation [22] can dilute the gravitino density and thus solve the gravitino problem. If $X_{I} \geq M_{\mathrm{P}}$ at the end of inflation, then such a second stage of inflation might naturally arise in our framework: once $H$ drops below the value of $m_{X}$ and the condensate can start to move, it will initially be slowly rolling and thus generate a second period of inflation.

Another consequence of the enhanced reheating scenario discussed here concerns the origin of cosmological fluctuations. As is well known quantum fluctuations of the $X$ condensate will lead to isocurvature fluctuations as in the case of axions (see, e.g., [23]) which upon the decay of the condensate will convert to adiabatic fluctuations via the curvaton mechanism [24]. However, the $X$ fluctuations will also lead to a spatial modulation of the reheating rate on super-Hubble scales and will thus generate fluctuations via the modulated decay mechanism [25] (this has already been pointed out in the context of the MSSM in [26]. As discussed in [27], the fluctuations which are generated in this way are primordial isocurvature fluctuations, and their production is completely consistent with causality.

The situation will be different when

$$
\Gamma<m_{X},
$$

$X_{I}<M_{\mathrm{P}}$, and if the parametric resonance channels for enhanced reheating are not open. In this case, the $X$ field starts oscillating at $H=m_{X}$, before reheating completes. From then on, $\langle X\rangle \propto H$ leading to $\Gamma_{I}(t) \propto H^{2}$. This implies that reheating will remain inefficient until $\Gamma_{d}$ settles at its value in the minimum $\Gamma_{0}$. The reheat temperature of the Universe will then be $T_{R, 0}$.

An important issue to take into consideration is early oscillations of $X$ due to thermal effects from perturbative reheating [28]. The $X$ field has gauge and/or Yukawa couplings (collectively denoted by $h$ ) to other fields. If $h X_{I} \leq T$, these degrees of freedom will be in equilibrium with the instantaneous thermal bath from reheating. This in turn induces a thermal mass $h T$ for the $X$ field which will trigger its early oscillations if $h T \geq H$. Early oscillations, if they start before reheating completes, would render the reheating inefficient as noted above. There will be no early oscillations if $h X_{I}>T$ or $h T<H$. This requires sufficiently large or small values of $h$, respectively, which can naturally occur for the MSSM flat directions and right-handed sneutrinos, respectively. The early oscillations will not be important in the case of parametric reheating. In this case, fields which are coupled to $X$ via gauge and/or Yukawa couplings reach thermal equilibrium on time scales much longer than that of the resonant inflaton decay [14].

\section{CONCLUSIONS}

In this Letter we discussed enhanced reheating channels which arise as a consequence of the presence of scalar condensate fields which acquire a large expectation value $X_{I}$ during inflation. The scalar condensates can result in large effective couplings of the inflaton to matter, 
which can enhance both the perturbative decay rate and the efficiency of parametric resonance instabilities. We have seen that efficient reheating is therefore possible even if inflaton has only gravitational couplings to matter fields in the bare Lagrangian.

Enhanced reheating can have many consequences for cosmology. Generically, the reheating temperature will be higher than what would result in the absence of the condensates. In supersymmetric models this can make the gravitino problem worse.

Since scalar condensates acquire fluctuations during inflation which are super-Hubble scale at the end of inflation, they provide a realization of the modulated fluctuation scenario of [25]: at the time of reheating, super-Hubble-scale entropy perturbations are generated as a consequence of the space-dependent effective coupling constants. This will relax the bounds from density perturbations on the inflaton mass $m_{\phi}$.

In summary, scalar condensates can lead to a successful scenario of chaotic inflation where the inflaton has only gravitational couplings and $m_{\phi} \ll 10^{13} \mathrm{GeV}$. Immediately after the phase of slow-roll inflation comes to an end and the inflaton field begins to oscillate, the large amplitude of the condensate $X_{I}$ leads to large effective couplings in the dimension 5 and 6 operators which couple the inflaton, the condensate, and matter fields. This opens up channels for decay of the inflaton via broad parametric resonance which would be closed in the absence of $X$. Thus, rapid reheating takes place. The decay will produce massive particles which can provide the dark matter and/or (in the case of right-handed neutrinos) the lepton asymmetry required for leptogenesis. At the same time, the super-Hubble-scale fluctuations of the condensate lead to isocurvature fluctuations both of an axionic type and of the type generated through modulated decay. A detailed study of enhanced reheating and its consequences for baryogenesis and dark matter will appear in a separate publication.

\section{ACKNOWLEDGMENTS}

The research of R. A. and A. M. is supported by the National Sciences and Engineering Research Council of Canada. A. M. is supported by CITA. R. B. is supported in part by the U.S. Department of Energy under Contract No. DE-FG02-91ER40688, TASK A. He thanks the Perimeter Institute for their gracious hospitality and financial support during the course of the work on this project. A. M. thanks the ASPEN center for physics for their kind hospitality during the course of this work.
[1] C. L. Bennett et al., Astrophys. J. Suppl. Ser. 148, 1 (2003).

[2] A. D. Linde, Particle Physics and Inflationary Cosmology (Harwood, Chur, Switzerland, 1990).

[3] D. H. Lyth and A. Riotto, Phys. Rep. 314, 1 (1999).

[4] H. P. Nilles, Phys. Rep. 110, 1 (1984).

[5] K. Enqvist and A. Mazumdar, Phys. Rep. 380, 99 (2003); M. Dine and A. Kusenko, Rev. Mod. Phys. 76, 1 (2004).

[6] A. Kusenko and M. E. Shaposhnikov, Phys. Lett. B 418, 46 (1998); G. R. Dvali, A. Kusenko, and M. E. Shaposhnikov, Phys. Lett. B 417, 99 (1998); K. Enqvist and J. McDonald, Phys. Lett. B 425, 309 (1998); Nucl. Phys. B538, 321 (1999).

[7] K. Enqvist, S. Kasuya, and A. Mazumdar, Phys. Rev. Lett. 90, 091302 (2003); K. Enqvist, A. Jokinen, S. Kasuya, and A. Mazumdar, Phys. Rev. D 68, 103507 (2003); K. Enqvist, S. Kasuya, and A. Mazumdar, Phys. Rev. Lett. 93, 061301 (2004); K. Enqvist, A. Mazumdar, and A. Perez-Lorenzana, hep-th/0403044.

[8] K. A. Olive, G. Steigman, and T. P. Walker, Phys. Rep. 333, 389 (2000).

[9] A. D. Dolgov and A. D. Linde, Phys. Lett. 116B, 329 (1982); L. F. Abbott, E. Farhi, and M. B. Wise, Phys. Lett. 117B, 29 (1982).

[10] J. H. Traschen and R. H. Brandenberger, Phys. Rev. D 42, 2491 (1990).
[11] A. D. Dolgov and D. P. Kirilova, Sov. J. Nucl. Phys. 51, 172 (1990) [Yad. Fiz. 51, 273 (1990)].

[12] L. Kofman, A. D. Linde, and A. A. Starobinsky, Phys. Rev. Lett. 73, 3195 (1994).

[13] Y. Shtanov, J.H. Traschen, and R. H. Brandenberger, Phys. Rev. D 51, 5438 (1995).

[14] G. N. Felder and L. Kofman, Phys. Rev. D 63, 103503 (2001).

[15] L. Kofman, A. D. Linde, and A. A. Starobinsky, Phys. Rev. D 56, 3258 (1997).

[16] P. B. Greene and L. Kofman, Phys. Lett. B 448, 6 (1999).

[17] V.F. Mukhanov, H. A. Feldman, and R. H. Brandenberger, Phys. Rep. 215, 203 (1992).

[18] M. Dine, L. Randall, and S. Thomas, Phys. Rev. Lett. 75, 398 (1995).

[19] M. Postma and A. Mazumdar, J. Cosmol. Astropart. Phys. 01 (2004) 005.

[20] M. Y. Khlopov and A. D. Linde, Phys. Lett. 138B, 265 (1984); J. R. Ellis, J. E. Kim, and D. V. Nanopoulos, Phys. Lett. 145B, 181 (1984); J. R. Ellis, D. V. Nanopoulos, K. A. Olive, and S. J. Rey, Astropart. Phys. 4, 371 (1996); for discussions of nonperturbative decay, see A. L. Maroto and A. Mazumdar, Phys. Rev. Lett. 84, 1655 (2000); R. Kallosh, L. Kofman, A. D. Linde, and A. Van Proeyen, Phys. Rev. D 61, 103503 (2000). 
[21] G. F. Giudice, M. A. Luty, H. Murayama, and R. Rattazzi, J. High Energy Phys. 12 (1998) 027.

[22] D. H. Lyth and E. D. Stewart, Phys. Rev. D 53, 1784 (1996).

[23] M. Axenides, R. H. Brandenberger, and M. S. Turner, Phys. Lett. 126B, 178 (1983).

[24] D. H. Lyth and D. Wands, Phys. Lett. B 524, 5 (2002).

[25] G. Dvali, A. Gruzinov, and M. Zaldarriaga, Phys. Rev. D 69, 023505 (2004); L. Kofman, astro-ph/0303614; F. Vernizzi, Phys. Rev. D 69, 083526 (2004).
[26] K. Enqvist, A. Mazumdar, and M. Postma, Phys. Rev. D 67, 121303 (2003); R. Allahverdi, Phys. Rev. D 70, 043507 (2004).

[27] F. Finelli and R. H. Brandenberger, Phys. Rev. Lett. 82, 1362 (1999); Phys. Rev. D 62, 083502 (2000).

[28] R. Allahverdi, B. A. Campbell, and J. Ellis, Nucl. Phys. B579, 355 (2000); A. Anisimov and M. Dine, Nucl. Phys. B619, 729 (2001). 